\title{
Quantum of fluids in hospitalised patients with dengue fever \\ and the presence of warning signs: a pilot cross-sectional
}

\section{study [version 1; peer review: 1 approved with reservations, 1}

\section{not approved]}

\author{
Ramayee Ramanathan1, Dheeraj Jain'1, Stalin Viswanathan (iD2 \\ ${ }^{1}$ Department of General Medicine, Indira Gandhi Medical College \& Research Institute, Pondicherry, Puducherry, 605009, India \\ ${ }^{2}$ Department of General Medicine, Jawaharlal Institute of Postgraduate Medical Education and Research (JIPMER)., Pondicherry, \\ Puducherry, 605009, India
}

\begin{tabular}{l}
\hline V1 First published: 05 Mar 2019, 8:260 \\
https://doi.org/10.12688/f1000research.18203.1 \\
Latest published: 15 May 2019, 8:260 \\
https://doi.org/10.12688/f1000research.18203.2 \\
\hline
\end{tabular}

\section{Abstract}

Background: The World Health Organization in 2009 revised categories of dengue fever severity into classes $A, B$, and $C$ based on the presence or absence of warning signs. The quantum of oral and intravenous fluids (IVF) in hospitalised patients with Group B (warning signs) have, to our knowledge, not been studied. Oral fluids in hospitalised patients and monitoring administration with the help of patients' relatives have not been assessed.

Methods: Consecutive patients with dengue fever and warning signs were studied for 48 hours after hospitalisation. Patients were asked to consume 4-5 I of fluids. Maintenance and bolus IVF were administered depending upon the presence of compensated or hypotensive shock. Intake and urinary output were monitored by the patient's attendant. Total fluids (oral and intravenous) were divided by a number of anthropometric measurements. Significance of compensated and hypotensive shock, thrombocytopenia and $20 \%$ change in haematocrit, bleeding episodes and the need for transfusions, and organ impairment were considered in relation to the total amount of fluids received daily.

Results: In total, 41 patients were studied. Patients with obesity and hypotensive shock received significantly more fluids on Day 1 ; taller patients and those with tachycardia, higher haematocrit and elevated creatine kinase correspondingly received more on Day 2. Hypotensive shock was significantly correlated with fluids/BSA and fluids/weight, while laboratory parameters correlated most strongly with fluids/BMI. Conclusions: Most adults with dengue and warning signs seem to require $>100 \mathrm{ml} / \mathrm{kg} /$ day of fluids during their stay. Advising a similar amount of fluids at home during epidemics may further reduce the need for admissions. Participation of patients and their relatives in the

\section{Open Peer Review}

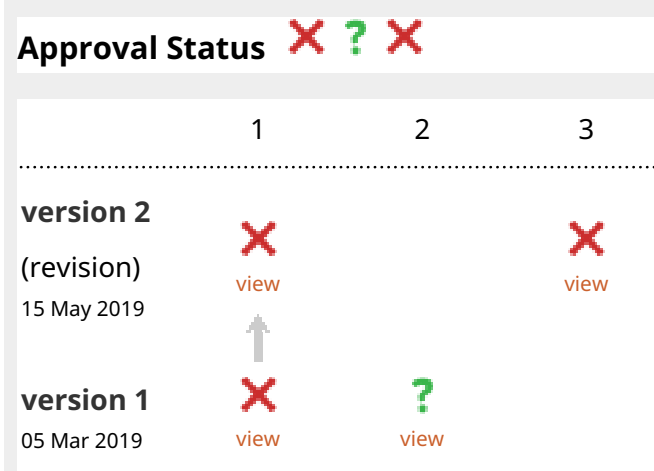

1. Siripen Kalayanarooj, Queen Sirikit National Institute of Child Health (QSNICH), Bangkok, Thailand

2. Anon Srikiatkhachorn (ID), University of Rhode Island, Providence, USA

3. Suranjith L. Seneviratne (D), Royal Free London NHS Foundation Trust, London, UK Any reports and responses or comments on the article can be found at the end of the article. 
bedside management of fluid administration may go a long way in preventing morbidity and mortality. Adults probably need a better anthropometric measurement to decide on the quantum of fluids to be given.

Keywords

dengue, fluid requirements, warning signs, guidelines, Pondicherry outbreak

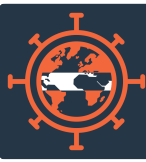

This article is included in the Neglected Tropical

Diseases collection.

Corresponding author: Stalin Viswanathan (stalinviswanathan@ymail.com)

Author roles: Ramanathan R: Data Curation, Investigation, Writing - Original Draft Preparation; Jain D: Conceptualization, Project Administration, Supervision; Viswanathan S: Conceptualization, Formal Analysis, Supervision, Writing - Review \& Editing

Competing interests: No competing interests were disclosed.

Grant information: The author(s) declared that no grants were involved in supporting this work.

Copyright: $@ 2019$ Ramanathan R et al. This is an open access article distributed under the terms of the Creative Commons Attribution License, which permits unrestricted use, distribution, and reproduction in any medium, provided the original work is properly cited.

How to cite this article: Ramanathan $R$, Jain $D$ and Viswanathan $S$. Quantum of fluids in hospitalised patients with dengue fever and the presence of warning signs: a pilot cross-sectional study [version 1; peer review: 1 approved with reservations, 1 not approved] F1000Research 2019, 8:260 https://doi.org/10.12688/f1000research.18203.1

First published: 05 Mar 2019, 8:260 https://doi.org/10.12688/f1000research.18203.1 


\section{Introduction}

Dengue fever is an acute arboviral febrile illness transmitted by Aedes mosquitoes and has four serotypes: DENV1, DENV2, DENV3, and DENV4'. It has been reported in over 70 countries and is endemic in the tropics, where $\geq 2.5$ billion people are at risk $^{2-4}$. India is a category A endemic country ${ }^{2}$; in such countries dengue strains the already over-stretched health care system, and increases the economic burden due to hospitalization, absence from school, loss of productivity, loss of tourism revenue, and out-of-pocket expenses ${ }^{2,4}$. Even this burden is a gross underestimate due to underreporting of cases and deaths, poor networking of healthcare facilities, and interstate/inter-regional differences in the availability of optimal healthcare ${ }^{5}$. The number of reported dengue cases in Pondicherry, South India had fallen from $>3500$ in 2012 to $<500$ in the year 2016 , with a reported total mortality of just six cases during those five years ${ }^{6}$.

Guidelines by the World Health Organization (WHO) have been used to manage patients with dengue for $\sim 45$ years ${ }^{7}$. Until 2009, dengue cases were classified as dengue fever, dengue haemorrhagic fever and dengue shock syndrome, and appropriate management protocols were prescribed by WHO and national guidelines ${ }^{8}$. The revised classification by severity categorised patients into dengue fever without warning signs (Class A), with warning signs (Class B), and those with severe dengue $(\text { Class } \mathrm{C})^{8}$. The warning signs include abdominal pain or tenderness, hepatomegaly, persistent vomiting, clinical fluid accumulation, mucosal bleed, lethargy and restlessness, and increase in haematocrit associated with fall in platelet counts. Patients in Group B must be admitted for the administration of fluids and observation during the critical phase, while patients in Group $\mathrm{C}$ should receive emergency care and/or urgent referral. Volume replacement guidelines by the WHO have been based on studies in children'. The quantum of fluids to be administered to patients in Group B have not been studied so far. Previously, only two studies had described the trends in fluid requirements over the patients' entire hospital stay but using the earlier dengue severity classification ${ }^{10,11}$. Two other studies, one each from Malaysia and Nicaragua, had described the effectiveness of drinking one litre of fluid before hospital admission ${ }^{12,13}$.

Even though only patients in class $\mathrm{B}$ and $\mathrm{C}$ require admission, most patients with dengue fever are often admitted due to inability to differentiate it from other acute febrile illnesses or from non-severe dengue itself ${ }^{9}$. Guideline-based administration of intravenous fluids (IVF) are based on patients' weight and are generally a step-down regimen as the patient's clinical and laboratory parameters improve. This is often not practical in primary care due to the shortage of doctors and nurses to monitor as prescribed in the guidelines, spacing constraints, and availability of 24-hour laboratory services. Surveillance for severe dengue is generally poor in countries with epidemics. This often leads to overcrowding at hospitals and clinics with dengue fever/mild illness. In association with overworked medical personnel and confusion among the lay public, this overcrowding often predisposes to less-than-optimal care for patients with severe dengue and increased mortality ${ }^{4}$.
In view of such a scenario, we decided to study patients hospitalized with dengue warning signs, wherein, they could be monitored and administered fluids, even with a low doctor/nurse-to-patient ratio, by informing patients in advance what their daily fluid intake was expected to be and using the assistance of their relatives in measuring fluid intake and output. This study aimed to find out the quantity of fluids adult patients with dengue fever and warning signs received to maintain hemodynamic stability and to discern which anthropometric measurement-based fluid requirements correlated best with manifestations of severe dengue.

\section{Methods}

\section{Study setting}

The study was conducted in Pondicherry during the 2017 dengue epidemic. All consecutive adolescent and adult patients admitted to Unit 3 of the Department of General Medicine, Indira Gandhi Medical College \& Research Institute, with a confirmed diagnosis of dengue fever between 11 October 2017 and 08 November 2017 were recruited for the study. Ethics approval was obtained from the Institute Ethics Committee to obtain and record data from all febrile patients (IEC/PP/2016/44). Written informed consent was obtained from all participants to use their clinical and laboratory data.

Patients with probable/confirmed dengue fever were admitted in the male medical ward (MMW), female medical ward (FMW), and a newly created dengue ward (DW). A total of 15 beds each had been added to the existing strength of FMW (60 beds) and MMW (70 beds) for ease of providing care. When these wards became full, newer patients were admitted into the DW (30 beds), which had a nurse available on all shifts, but without additional residents or house surgeons. The MMW and FMW were staffed by three nurses in the morning shift and two each in the afternoon and night shifts. The DW had two nurses in the morning and one each in the other two shifts. Each Unit was allotted 26 beds (26/130). Two faculty members, three residents, and three house surgeons of Unit 3 oversaw treatment of these patients. All doctors worked from 8:30 AM to 4:30 PM on weekdays, and from 8:30 AM to 1:00 PM on weekends. House surgeons managed night ward duties on a daily rotation, while residents stayed nights once a week. Patients could have one attendant/relative stay with them in the ward as part of hospital policy.

\section{Patients}

All patients 13 years or older examined in the outpatient or emergency department or with an acute febrile illness and a probable diagnosis of dengue fever were considered for the study. Such patients underwent a focussed clinical examination at the time of admission that included vitals, gums, skin, pallor, jugular venous pulse, oedema, hepatosplenomegaly, respiratory auscultation for crackles and diminished breath sounds. A complete blood count, liver function tests, renal function tests, creatine kinase, dengue IgM and NS1, urinalysis, chest radiograph, and electrocardiogram (ECG) were performed as part of routine care for febrile patients with suspected dengue. Patients were shifted to the wards after being stabilized in the emergency 
department. Those confirmed to have dengue fever by either dengue IgM or NS1 antigen positivity and had had warning signs at admission were considered eligible, and informed consent sought was obtained for inclusion in the study. Patients unwilling to participate, patients having dengue without warning signs, and those with an alternative diagnosis for the cause of acute febrile illness were excluded. Day 1 began at 8:00 AM on the following day after admission, and all patients were monitored for 48 hours until 8:00 AM of Day3.

All patients had been admitted into the hospital because of one or more of the following warning signs: persistent vomiting, abdominal pain, poor oral intake, dizziness, hypotension or shock, tachycardia during the afebrile phase of illness, severe thrombocytopenia, mucosal bleed, associated diabetes, pregnancy or alcoholism, and referral for admission from a peripheral health centre with poor transport facilities. Even though obese/overweight patients with difficult intravenous access are also considered as warning signs, we did not encounter difficulty in access and hence, they were not considered as a criterion for admission/warning $\operatorname{sign}^{8}$.

\section{Definitions}

Patients were classified into those with Class B dengue and those with Class $\mathrm{C}$, based on WHO guidelines ${ }^{8}$. Class $\mathrm{C}$ included those with serositis, compensated and hypotensive shock, dysfunction in one or more organs, severe bleeding requiring blood products, severe thrombocytopenia, and a change in haematocrit by $20 \%$. Postural symptoms were defined by the presence of either dizziness, light-headedness, feeling faint, palpitations, or sweating while sitting or standing from the supine position. Postural hypotension $(\mathrm{PH})$ was considered when the systolic BP fell $\geq 20 \mathrm{~mm}$ and/or the diastolic blood pressure (DBP) fell $\geq 10 \mathrm{~mm}$ when the patient stood upright for two minutes after getting up from the supine position. In those patients with postural symptoms, blood pressure was recorded in the sitting position with legs dangling down. Postural BP measurements were done at least twice daily and the higher fall in BP was noted. The postural BP in the morning was taken under the supervision of the consultant taking rounds, while the evening and night shift recordings were taken by either the duty resident or house surgeon. Hypotension was considered when the systolic blood pressure was $<100 \mathrm{mmHg}$. Tachycardia was when the pulse rate was $\geq 100$ beats/minute. The presence of either tachycardia or postural hypotension defined compensated shock. One of the following was considered as hypotensive shock: pulse pressure was $\leq 20 \mathrm{mmHg}$, systolic blood pressure (SBP) was $<90 \mathrm{mmHg}$, feeble pulse or the patient was restless. Serositis was defined by the new onset development of ascites and/ or pleural effusion. Organ impairment was defined by either/or the presence of acute kidney injury (Acute Kidney Injury Network criteria) ${ }^{14}$, transaminases elevation $\geq 3$ times (hepatic dysfunction), CK elevation $\geq 3$ times (myositis), and new onset cardiac arrhythmias. Patients were overweight when the body mass index (BMI) was $\geq 25 \mathrm{~kg} / \mathrm{m}^{2}$. Bleeding was defined by the presence of new bleeding from any site- gums, nose (epistaxis), gastrointestinal (hematemesis, melena or haematochezia), respiratory(haemoptysis), uterine (menorrhagia/ metrorrhagia) or skin (purpura or petechiae). Warning signs were defined by the presence of one or more of the following: abdominal pain, vomiting, dyspnea, dizziness, hepatomegaly, mucosal bleeding, diabetes mellitus, pregnancy, and alcoholism. A platelet count of $<150 \times 10^{9} / 1$ was defined as thrombocytopenia, while severe thrombocytopenia was when the counts were $<50 \times 10^{9} / 1$. A significant change in haematocrit from Day 1 to Day 2 was considered when there was either a $20 \%$ increase or fall in haematocrit.

\section{Observations and treatment}

Anthropometry (height, weight, BMI and body surface area) were measured on Day 1. BMI and body surface area (BSA) was calculated using the QxMD Calculate mobile app (version 7.0.6.0), using the De Bois formula. Pulse rate, SBP, DBP, pulse pressure, systolic postural fall (SBP fall) and diastolic postural fall (DBP fall) were measured at least twice daily during both days. More frequent measurements were done in those with postural symptoms and when receiving bolus IVF. Similarly, examination for abdominal tenderness, hepatomegaly, ascites, pleural effusion, altered consciousness were performed twice daily. Urea/ creatinine, LFT and CK-total were done once at admission and again 48 hours later to monitor trends. CBC was performed twice daily during the 48 hours for all patients, and every $6^{\text {th }}$ hourly in those with bleeding or $20 \%$ increase in haematocrit.

All patients were asked to drink 4-5 1 of oral fluids cumulatively (a combination of water with or without oral rehydration salt, water with 5 tsp glucose, fresh juice, tender coconut water, buttermilk, milk, and dilute millet or rice porridge). The patients and their relatives were instructed to keep five 1-1 bottles at the bedside to be filled up with water or oral rehydration salts each morning for easy monitoring. Other beverages were accounted as follows using two disposable paper cups of $200 \mathrm{ml}$ and $100 \mathrm{ml}$ each: a glass of juice, water with 5 tsp glucose, tender coconut water or porridge as $200 \mathrm{ml}$; 1 glass of buttermilk or milk as $100 \mathrm{ml}$. The total quantity of oral fluids was rounded-off to the nearest $100 \mathrm{ml}$. IVF was initiated for those in with postural symptoms, hypotension, shock, abdominal pain, persistent vomiting, or those with poor oral intake. Either normal saline or ringer lactate was administered as maintenance fluids, at the rate of $100 \mathrm{ml} /$ hour, calculated using the following formula: $\{1500 \mathrm{ml}+$ [weight $(\mathrm{kg}) \times 20 \mathrm{ml} / \mathrm{kg} /$ day] $=2500 \mathrm{ml}$, for a $50-\mathrm{kg}$ person $\}$. Boluses of normal saline $(500 \mathrm{ml})$ were given for those patients in shock and in those with significant postural fall in blood pressure. Dextrose saline (5\% DNS) was administered for patients with poor calorie intake and in those with hepatic dysfunction. The empty fluid bottles were retained at the patient's bedside cupboard to keep count of IVF to the nearest $500 \mathrm{ml}$. The attendants of patients who could document both oral and IVF intake on paper were exempted from keeping bottle counts. Fluid intake was measured at the end of Day 1 and Day 2. Urine output was measured every $6^{\text {th }}$ hourly. The total fluid counts (oral +intravenous) for each day was divided by each patient's weight, height, BMI and BSA. A measuring jar cut out from an IVF bottle, was used for measuring urine output (Figure 1). Attendants were instructed to inform and/or note down the colour and quantity of urine and urgently bring to notice if there was no urine output for six continuous hours. Outcome measures were the total oral fluids and total intravenous fluid 


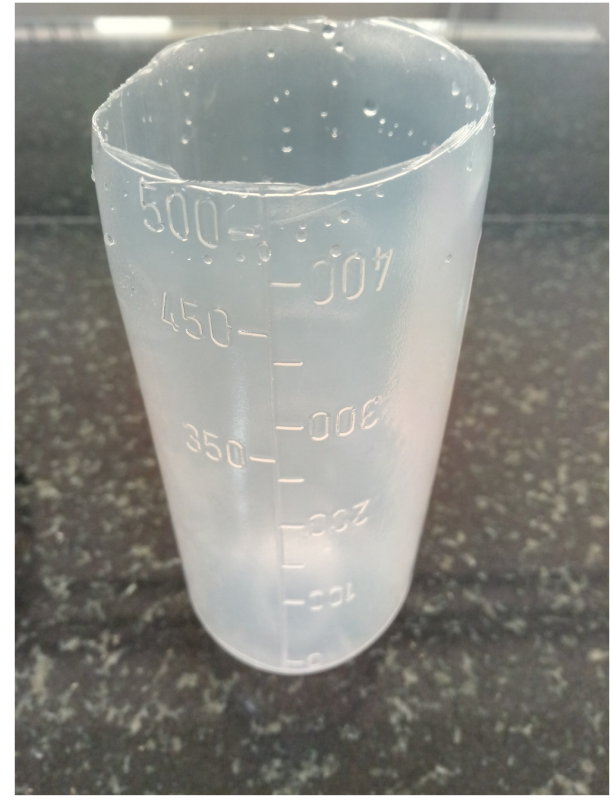

Figure 1. A crude container for measuring urinary volume.

administered, low urine output ( $<500 \mathrm{ml} /$ day), bleeding episodes and need for transfusion, compensated and hypotensive shock, and organ dysfunction such as hepatitis and myositis.

\section{Statistical analysis}

Data were entered in an Excel spreadsheet and thence imported into IBM SPSS for Windows v22 for statistical analysis. Frequencies of clinical symptoms, examination findings, and severe organ involvement were calculated. Chi-square analysis (Fischer's exact test for frequencies <5) was used to compare the presence of symptoms, signs, and investigations among those who had received more than 51 fluid/day with those who had not. Means \pm standard deviations were calculated for continuous variables (clinical, lab, and treatment) and compared between the two groups receiving and not receiving 51 fluids on both days using t-test. Bivariate correlations were performed between fluid administration (based on weight, height, BMI, and BSA on both days) and clinical/lab values. $\mathrm{P}<0.05$ was considered to indicate a statistically significant difference.

\section{Results}

Patient condition and fluid consumption

Of the 43 patients, 30 were female. Most patients were admitted on the fourth $(n=9)$, fifth $(n=8)$, and seventh $(n=7)$ days of fever. Males had a significantly higher postural fall compared to females and had higher haematocrit and CK values. At admission, all patients had had warning signs, while 37 continued to have warning signs on Day 1 , and on further evaluation and investigations, 33 of them also had signs of severe dengue (Class C) (Table 1). In total, 15 had serositis and/or organ involvement in the form of either hepatitis, AKI, myositis, or cardiac dysfunction ( 1 case of Brugada Type 1 ECG pattern). In total, 27 were afebrile on Day 1, and 36 on Day 2. BMI was comparable between males and females $(24.79 \pm 6.45$ versus 23.98 \pm 4.92 ). Thrombocytopenia was seen in 12 and 11 patients on Day 1 and Day 2, respectively, with bleeding manifestation in seven (three with gum bleeds and one each with epistaxis, metrorrhagia, haemoptysis and hematochezia). Only four patients had an increase in haematocrit $\geq 20 \%$ on Day 2 compared with the previous day. Obese individuals and patients with hypotensive shock received significantly more fluids on Day 1 than adolescents (Table 2). There was no significant difference in fluids received in those with and without warning signs. Bleeding was not significantly related to platelet counts, haematocrit, liver dysfunction, or CK elevation. Serositis, hepatitis, severe thrombocytopenia, bleeding, and compensated shock did not correlate with any anthropometry-related fluid calculation. Hypotensive shock had significant correlations with fluids/BSA and fluids/weight, while laboratory parameters correlated with best with fluids/BMI (Table 3). None of them developed fluid overload, pulmonary oedema, ARDS or admission into intensive care unit (ICU). There was no mortality. Raw data are available on Harvard Dataverse ${ }^{15}$.

\section{Patient attitude towards drinking fluids}

Patients' inability/hesitancy to drink fluids as instructed by the staff were due to poor taste, nausea, vomiting, abdominal pain, dizziness on sitting up or walking to the restroom, inability to use the restroom following increased fluid intake, and poor cleanliness of the common restrooms. Patients referred from peripheral health centres for admission also felt that care was incomplete without IVF, preferring IVF over oral fluids, and often demanded IVF. In those who received IVF, problems encountered include wrong beliefs that they should not eat or drink while receiving IVF, unavailability of IVF stands on occasions (due to requirement by most patients in the ward), thrombophlebitis, delay in nurse or a doctor reaching them to disconnect the IV line so that they could use the toilet and then reconnect it again, difficulty in sleeping in a comfortable position because of the cannula, and washing after using the toilet (IV cannula in the left wrist/hand).

\section{Discussion}

There are 50-100 million new cases of dengue annually, with the vast majority of them in individuals $>15$ years of age $\mathrm{a}^{4,16}$. Dengue began being reported in Pondicherry from 2007 onwards ${ }^{1}$. A study from a hospital in Pondicherry during 2017 described the peak incidence of dengue between September and October; the dengue seroprevalence among antenatal mothers and children with fever was found to be $25.8 \%^{17}$. Severe dengue is more common in children ${ }^{18}$. The mean age of patients with severe dengue in our study was $30 \pm 12$ years, compared to $26 \pm 11$ years in those without severe dengue. Reports from India show that confirmed cases are being described mostly in the 21-30 years age group ${ }^{1}$. Even though dengue shock requires admittance to an ICU, we managed all patients in the wards due to a shortage of beds in the $\mathrm{ICU}^{3}$. None of our patients developed other indications of ICU care such as ARDS, cardiogenic shock, encephalitis, or secondary pneumonia.

Fluid calculation during the critical phase is calculates as follows: $100 \mathrm{ml} / \mathrm{kg}$ for the first $10 \mathrm{~kg}(1000 \mathrm{ml}), 50 \mathrm{~mL} / \mathrm{kg}$ for 
Table 1. Clinical and lab values in patients who received $\geq \mathbf{5} \mathbf{l} / \mathbf{d}$ fluids on Day $\mathbf{1}$ and Day 2. Values are given as mean \pm standard deviation where indicated.

\begin{tabular}{|c|c|c|c|c|c|c|}
\hline Variables & $\begin{array}{l}<5 \text { L fluids on } \\
\text { Day } 1(n=18)\end{array}$ & $\begin{array}{l}\geq 5 L \text { fluids on } \\
\text { Day } 1(n=25)\end{array}$ & Significance & $\begin{array}{l}<5 L \text { fluids on } \\
\text { Day } 2(n=15)\end{array}$ & $\begin{array}{l}\geq 5 L \text { fluids on } \\
\text { Day } 2(n=28)\end{array}$ & Significance \\
\hline Age (Years \pm SD) & $28.2 \pm 14.6$ & $29.8 \pm 10.8$ & 0.67 & $33.8 \pm 13.1$ & $26.6 \pm 11.5$ & 0.06 \\
\hline Adolescents ( $n$ ) & 7 & 4 & 0.09 & 2 & 9 & 0.17 \\
\hline Fever duration (days \pm SD) & $6.33 \pm 1.49$ & $6.12 \pm 2.42$ & 0.74 & $7.07 \pm 2.57$ & $5.75 \pm 1.60$ & 0.045 \\
\hline Abdominal pain $(n)$ & 4 & 3 & 0.37 & 5 & 2 & 0.02 \\
\hline Dizziness (n) & 4 & 8 & 0.48 & 5 & 7 & 0.56 \\
\hline Hepatomegaly (n) & 5 & 8 & 0.76 & 5 & 8 & 0.76 \\
\hline Warning signs & 15 & 22 & 0.60 & 14 & 23 & 0.51 \\
\hline $\begin{array}{l}\text { ECG HR at admission } \\
\text { (beats/min } \pm \text { SD) }\end{array}$ & $90.4 \pm 15.3$ & $89.9 \pm 28.9$ & 0.94 & $76.7 \pm 28.1$ & $97.6 \pm 17.3$ & 0.04 \\
\hline Pulse Day 1 (Beat/min \pm SD) & $87.1 \pm 11.8$ & $89.1 \pm 14.6$ & 0.63 & $80.7 \pm 11.2$ & $87.6 \pm 11.0$ & 0.04 \\
\hline Compensated shock Day 1 (n) & 4 & 7 & 0.66 & 3 & 8 & 0.53 \\
\hline Hypotensive shock Day 1 (n) & 0 & 7 & 0.03 & 1 & 6 & 0.21 \\
\hline Pulse Day 2 (Beats/min $\pm S D)$ & $83.5 \pm 11.2$ & $86.0 \pm 11.9$ & 0.48 & $80.7 \pm 11.2$ & $87.6 \pm 11.0$ & 0.03 \\
\hline Compensated shock Day 2 (n) & 7 & 6 & 0.29 & 6 & 7 & 0.30 \\
\hline Hypotensive shock Day 2 (n) & 0 & 5 & 0.04 & 2 & 3 & 0.79 \\
\hline Weight (kg $\pm S D)$ & $54.4 \pm 13.4$ & $59.4 \pm 9.8$ & 0.17 & $55.1 \pm 11.6$ & $58.5 \pm 11.6$ & 0.37 \\
\hline Height $(m \pm S D)$ & $1.59 \pm 0.84$ & $1.57 \pm 0.65$ & 0.53 & $1.54 \pm 0.52$ & $1.60 \pm 0.74$ & 0.008 \\
\hline $\mathrm{BMI}\left(\mathrm{kg} / \mathrm{m}^{2} \pm \mathrm{SD}\right)$ & $22.4 \pm 6.2$ & $25.4 \pm 4.3$ & 0.06 & $24.4 \pm 5.2$ & $24.1 \pm 5.4$ & 0.84 \\
\hline Obesity (n) & 4 & 14 & 0.02 & 4 & 14 & 0.13 \\
\hline $\mathrm{BSA}\left(\mathrm{m}^{2} \pm \mathrm{SD}\right)$ & $1.5 \pm 0.1$ & $1.6 \pm 0.1$ & 0.21 & $1.5 \pm 0.1$ & $1.5 \pm 0.1$ & 0.17 \\
\hline HCT Day $1( \pm \mathrm{SD})$ & $37.2 \pm 6.8$ & $37.7 \pm 6.1$ & 0.80 & $34.1 \pm 6.9$ & $39.3 \pm 5.3$ & 0.008 \\
\hline HCT Day $2( \pm S D)$ & $36.0 \pm 5.3$ & $38.3 \pm 7.5$ & 0.80 & $34.0 \pm 5.2$ & $39.1 \pm 6.8$ & 0.01 \\
\hline Platelet Day $1\left(\times 10^{9} / \mathrm{L} \pm \mathrm{SD}\right)$ & $102.5 \pm 57.2$ & $113.6 \pm 60.0$ & 0.54 & $118.6 \pm 60.3$ & $103.8 \pm 57.9$ & 0.44 \\
\hline Platelet Day $2\left(\times 10^{9} / \mathrm{L} \pm \mathrm{SD}\right)$ & $99.6 \pm 71.4$ & $108.9 \pm 84.7$ & 0.70 & $120.2 \pm 102.9$ & $96.9 \pm 62.7$ & 0.36 \\
\hline Bleeding $(n)$ & 3 & 4 & 0.95 & 4 & 3 & 0.17 \\
\hline Hepatitis (n) & 0 & 3 & 0.12 & 1 & 2 & 0.95 \\
\hline SGOT Day 1 (U/L $\pm S D)$ & $121.5 \pm 79.5$ & $103.5 \pm 86.9$ & 0.57 & $83.91 \pm 55.4$ & $129.0 \pm 93.7$ & 0.12 \\
\hline SGPT Day $1(\mathrm{U} / \mathrm{L} \pm \mathrm{SD})$ & $50.2 \pm 47.3$ & $36.6 \pm 46.3$ & 0.35 & $50.1 \pm 52.1$ & $38.1 \pm 44.1$ & 0.43 \\
\hline CK total $(\mathrm{U} / \mathrm{L} \pm \mathrm{SD})$ & $452.4 \pm 393.9$ & $262.7 \pm 243.2$ & 0.16 & $112.1 \pm 85.1$ & $469.5 \pm 337.2$ & 0.001 \\
\hline Myositis (n) & 4 & 4 & 0.60 & 0 & 8 & 0.005 \\
\hline
\end{tabular}

ECG, electrocardiogram; HR, heart rate; BMI, body mass index; BSA, body surface area; SGOT, serum glutamic oxaloacetic transaminase; SGPT, serum glutamic pyruvic transaminase; CK, creatine kinase.

the second $10 \mathrm{~kg}(500 \mathrm{ml})$ and $20 \mathrm{ml} / \mathrm{kg}$ for $>20 \mathrm{~kg}$ up to $50 \mathrm{~kg}$ $(600 \mathrm{ml})$, and $5 \%$ deficit, calculated as $50 \mathrm{ml} / \mathrm{kg}$ up to $50 \mathrm{~kg}$ $(2500 \mathrm{ml})$ which yields a total of $4600 \mathrm{ml}$ for a $50 \mathrm{~kg}$ person for about 48 hours $^{19}$. Since $33 / 37$ patients had severe dengue, the fluids administered were much higher than this amount during each day of the two-day study. Some studies have shown that the new WHO guidelines increased the sensitivity of identifying patients with severe illness, with a risk of over-admission and increased workload, which would not be desirable in endemic regions that were resource-limited ${ }^{7}$. In a study from a tertiary hospital at Singapore, some cases of dengue with warning signs were safely managed in outpatient settings, but this practice cannot be extrapolated to other settings of primary/secondary care $^{7}$. Most patients with severe illness progressed within a day of developing warning signs ${ }^{7}$. Thus, our study attempted to find out whether a range of fluid requirement would satisfy most patients (at least in a hospital) with dengue and warning signs without the minute assistance of a doctor or a nurse.

Only four studies have quantified the fluid requirements of patients with dengue, among which two have been performed in hospitalised patients ${ }^{10-13}$. Three had used the earlier WHO classification for severity of dengue: dengue fever, dengue haemorrhagic fever and dengue shock syndrome. The actual requirements of fluids in these three subtypes have not been quantified and vary with individuals; it depends on the 
Table 2. Fluid requirements on Day 1 and Day 2. Values are given as mean \pm standard deviation.

\begin{tabular}{|c|c|c|c|c|c|c|}
\hline & \multicolumn{3}{|c|}{ Fluids on Day 1 (ml) } & \multicolumn{3}{|c|}{ Fluids on Day 2 (ml) } \\
\hline Oral fluids (mean $\pm S D$ ) & \multicolumn{3}{|l|}{$3834.88 \pm 1872.15$} & \multicolumn{3}{|l|}{$4673.33 \pm 2101.31$} \\
\hline Intravenous fluids (mean \pm SD) & \multicolumn{3}{|l|}{$2318.60 \pm 1170.11$} & \multicolumn{3}{|l|}{$1702.33 \pm 1263.11$} \\
\hline Total fluids (mean \pm SD) & \multicolumn{3}{|l|}{$6141.86 \pm 2448.20$} & \multicolumn{3}{|l|}{$6433.72 \pm 2793.37$} \\
\hline Variables (Yes /No) & Yes & No & Significance & Yes & No & Significance \\
\hline Male gender $(n=13)$ & $7134.6 \pm 2848.9$ & $5711.6 \pm 2165.3$ & 0.08 & $8700 \pm 2585.9$ & $5451.6 \pm 2333.1$ & $<0.001$ \\
\hline Adolescents $(n=11)$ & $5086.3 \pm 1824.0$ & $6504.6 \pm 2552.6$ & 0.09 & $7909.0 \pm 2539.4$ & $5926.5 \pm 2730.1$ & 0.04 \\
\hline Obese $(n=18)$ & $7044.4 \pm 2822.4$ & $5492.0 \pm 1948.1$ & 0.03 & $7136.1 \pm 3173.0$ & $5929.0 \pm 2426.3$ & 0.16 \\
\hline Warning signs $(n=37)$ & $6108.5 \pm 2468.1$ & $6287.5 \pm 2518.7$ & 0.85 & $6361.4 \pm 2838.7$ & $6750.0 \pm 2745.3$ & 0.72 \\
\hline Compensated Shock $1(n=11)$ & $6368.1 \pm 1924.8$ & $6064.0 \pm 2626.9$ & 0.72 & $6354.5 \pm 2819.8$ & $6460.9 \pm 2819.8$ & 0.91 \\
\hline Hypotensive shock1 ( $n=7)$ & $8035.7 \pm 2817.0$ & $5773.6 \pm 2230.5$ & 0.02 & $7571.4 \pm 3526.9$ & $6212.5 \pm 2630.9$ & 0.24 \\
\hline Compensated shock2 $(n=13)$ & $6053.8 \pm 2901.9$ & $6180.0 \pm 2278.3$ & 0.87 & $6407.6 \pm 3858.4$ & $6445.0 \pm 2267.1$ & 0.96 \\
\hline Hypotensive Shock $2(n=5)$ & $8400.0 \pm 1763.5$ & $5844.7 \pm 2384.8$ & 0.02 & $5290.0 \pm 2908.2$ & $6584.2 \pm 2782.6$ & 0.33 \\
\hline Serositis $(n=10)$ & $5630.0 \pm 2205.0$ & $6296.9 \pm 2528.3$ & 0.72 & $6160.0 \pm 1435.4$ & $6516.7 \pm 3103.4$ & 0.43 \\
\hline Bleeding $(n=7)$ & $6921.4 \pm 2250.1$ & $5990.2 \pm 2485.8$ & 0.36 & $5078.5 \pm 2143.9$ & $6697.2 \pm 2852.5$ & 0.16 \\
\hline Hepatitis $(n=3)$ & $6100.8 \pm 871.8$ & $6145.0 \pm 2532.9$ & 0.97 & $8333.3 \pm 3547.2$ & $6291.2 \pm 2731.2$ & 0.22 \\
\hline Myositis $(n=8)$ & $6450.0 \pm 2332.2$ & $6071.4 \pm 2501.3$ & 0.69 & $8062.5 \pm 1298.2$ & $6061.4 \pm 2919.7$ & 0.06 \\
\hline
\end{tabular}

Table 3. Significant correlations of severe dengue manifestations with anthropometry-related fluid calculations.

\begin{tabular}{|l|l|l|l|l|l|}
\hline & $\begin{array}{l}\text { Fluids/BSA } \\
\text { Day 1 }\end{array}$ & $\begin{array}{l}\text { Fluids/Weight } \\
\text { Day 1 }\end{array}$ & $\begin{array}{l}\text { Fluids/BMI } \\
\text { Day 2 }\end{array}$ & $\begin{array}{l}\text { Fluids/BSA } \\
\text { Day 2 }\end{array}$ & $\begin{array}{l}\text { Fluids/Weight } \\
\text { Day 2 }\end{array}$ \\
\hline Hypotensive Shock Day 1 & 0.320 & & & & \\
\hline Hypotensive Shock Day 2 & 0.385 & 0.378 & & & \\
\hline Haematocrit Day 1 & & & 0.372 & 0.330 & 0.404 \\
\hline Haematocrit Day 2 & & 0.463 & 0.477 & 0.455 \\
\hline Haematocrit 20\% increase & & -0.311 & -0.311 & \\
\hline SGOT & & 0.410 & & \\
\hline CK total & & 0.562 & 0.507 & 0.613 \\
\hline Myositis & & 0.366 & & 0.405 \\
\hline
\end{tabular}

BSA, body surface area;SGOT, serum glutamic oxaloacetic transaminase; CK, creatine kinase.

availability of resources, staff, and place of treatment. Two studies had reported fluid requirements from admission to discharge $\mathrm{e}^{10,11}$. A large prospective study of 1501 patients in Asia and Latin America with children constituting $65 \%$ described fluid accumulation, shock, and respiratory distress in relation to IV fluids, especially boluses. Median IVF volumes for maintenance, rehydration, and resuscitation were 50, 50 and $64.71 / \mathrm{kg}$, respectively. The quantity of oral fluids consumed was not reported ${ }^{11}$. Another Sri Lankan study had shown that the quantity of fluid consumed had no impact on the development of third space fluid accumulation'. We are the first to assess fluid (oral and IVF) requirements based on the severity classification of $\mathrm{A}, \mathrm{B}$, and $\mathrm{C}$. We had practical difficulties with both oral and
IVF (as listed under Results), but persisted in explaining the need for fluids, especially by mouth.

Fluids losses into the third spaces may occur even prior to the fourth day of fever and it is imperative that fluid replacement begins early, especially prior to admission, beginning at home ${ }^{10}$. Our study showed that the maximum total fluids $(13,700$ and $14,100 \mathrm{ml}$ on Day 1 and Day 2, respectively) had been administered on the fourth day of fever (Figure 2). During the first two days of fever, it is difficult to predict whether the disease may progress to shock (compensated or hypotensive) and hence fluid supplementation is important. In the Sri Lankan study auditing the trends in fluid requirements, $6267 \mathrm{ml}$ was the mean 

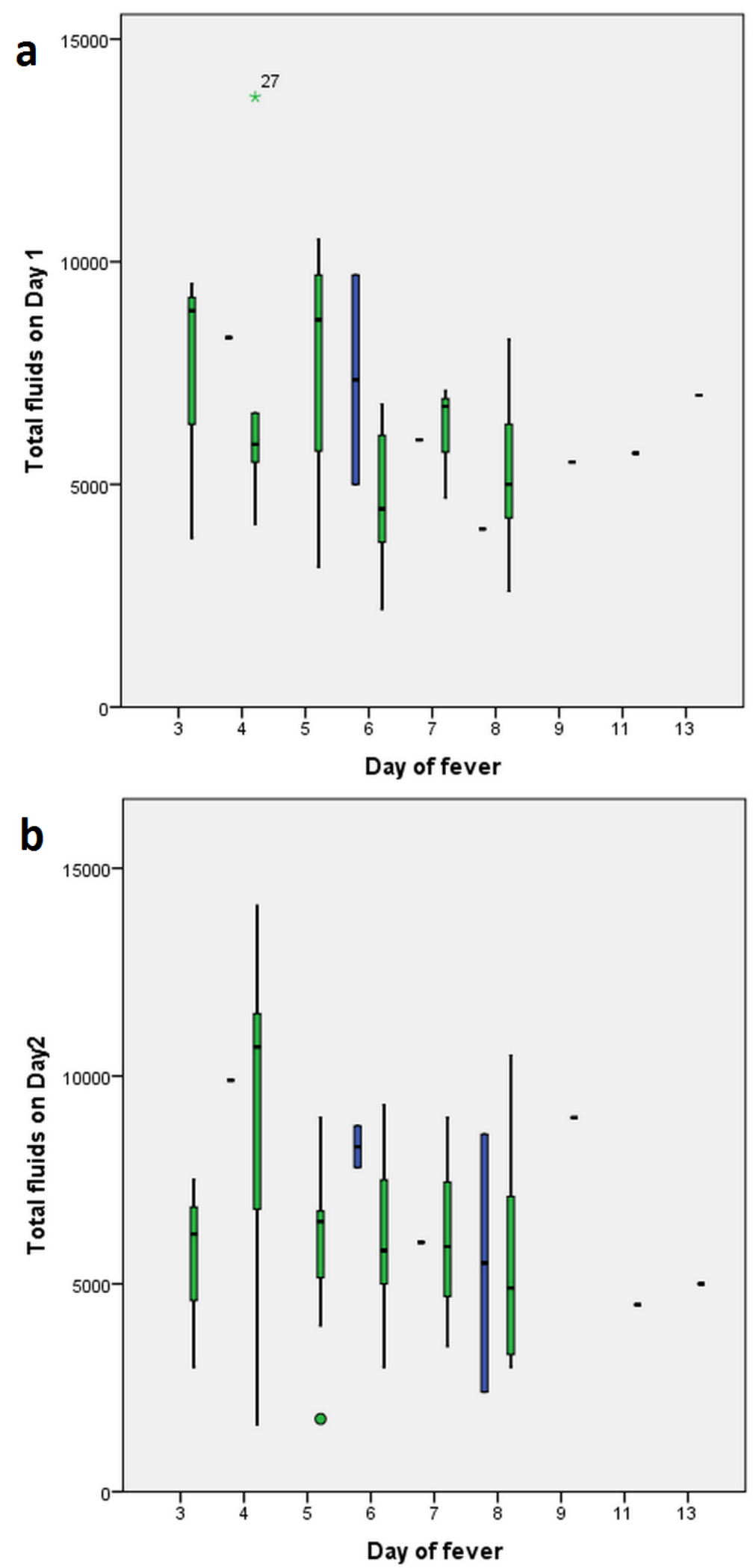

Figure 2. Box-plot showing fluids received on the two days of the study. 
requirement on the fourth day of fever similar to that of our study $(6141 \mathrm{ml} \text { on Day } 1 \text { and } 6433 \mathrm{ml} \text { on Day } 2)^{10}$.

The Malaysian study showed that reading out advice from a dengue home care card to patients attending primary care clinics improved fluid intake by at least one litre and reduced hospitalization rates ${ }^{12}$. They also found that a fluid chart maintained by the patient improved awareness of fluid intake and enhanced compliance. In Nicaragua, where the amount of fluid was also quantified, drinking five glasses of fluids prior to being seen by a doctor was protective against hospitalization ${ }^{13}$. The various liquids ingested in that study included juices, lemonade, milk, coffee, tea, and oral rehydration salts. We did not advise tea or coffee because of their weak diuretic action. We also suggested tender coconut water, dilute millet or rice porridge. Promoting high fluid intake was suggested to make an economic impact in countries with dengue fever ${ }^{13}$. We suggested an empirical 4-5 litre count based on the $100 \mathrm{ml} / \mathrm{kg}$ for an average $50 \mathrm{~kg}$ person, to account for both maintenance and replacement fluids arising from losses due to vomiting, diarrhoea, high fever, anorexia and poor intake in hot and humid weather conditions.

Volume replacement guidelines are based on studies in children and hence caution was advised in order to avoid fluid overload ${ }^{16}$. Intravenous fluids were given at a mean volume of $110 \mathrm{ml} / \mathrm{kg}$ over a mean of 25.3 hours in infants in a Vietnamese study which studied both patients with dengue hemorrhagic fever and dengue shock syndrome ${ }^{16}$. This contrasted with a mean IVF volume of $40.44 \mathrm{ml} / \mathrm{kg}(2318.6 \mathrm{ml} / 57.33 \mathrm{~kg})$ on Day 1 and $29.69 \mathrm{ml} / \mathrm{kg}(1702.33 \mathrm{ml} / 57.33 \mathrm{~kg})$ on Day 2 in our study. Hence the requirement of administered fluids is much lower when considered for their weights. A different anthropometric measurement may perhaps be useful in adults to gauge actual requirements, since the BSA and BMI correlated better with laboratory parameters of severe dengue in our study. Fluids/BSA correlated best with hypotensive shock on both days, while fluids/BMI correlated significantly with all the lab predictors of severe dengue.

\section{Limitations}

This study had very small numbers due to the fact it was an audit of treatment practices in a single treating unit in Medicine (total of five units) during an epidemic that lasted 2 months. The dengue species was not known. The blood pressure was measured using a mercury sphygmomanometer, where errors in a few millimetres could probably misclassify patients with compensated and hypotensive shock. Also, some patients with severe postural symptoms had their BP checked in the sitting position, which could have led to a higher pulse pressure being recorded. The ECG heart rate was documented only at admission and patients could have been misclassified as having or not having a warning sign during Day 1 of the study. For logistic reasons, the study began only at $0800 \mathrm{AM}$ of the following day after admission and hence the fluid administration for patients with shock or warning signs during the previous hours was not quantified. This amount may have been higher since all patients had been admitted initially with warning signs.

\section{Conclusions}

Most adults hospitalised with dengue fever and warning signs seem to require $>100 \mathrm{~mL} / \mathrm{kg} /$ day of fluids during their hospital stay without developing symptoms of fluid overload. This is over and above what has been described in the national guidelines, which are weight-based calculations. Abdominal pain, overweight individuals, tachycardia on ECG, hypotensive shock, increasing haematocrit and elevated CK levels were significantly associated with having received $>5$ 1/day. Since some patients developed warning signs on the third day of fever, it would be prudent to sometimes lower the threshold for admission in patients who come to the emergency department. Advising a larger amount of fluids (as in our study) at home during dengue epidemics could probably further reduce the need for admissions and/or development of severe dengue- a fraction of the fluid quantity that we administered our patients had already shown to reduce hospitalization rates in two other studies. In resource-poor settings, letting patients and their attendants participate in the acute bedside management of dengue with warning signs may go a long way in preventing morbidity in dengue fever.

\section{Data availability}

Harvard Dataverse: Quantum of fluids in hospitalised patients with dengue and warning signs- a pilot cross-sectional study. https:// doi.org/10.7910/DVN/SKEJAM ${ }^{15}$.

This project contains information on the fluids consumed and patient blood values.

Data are available under the terms of the Creative Commons Zero "No rights reserved" data waiver (CC0 1.0 Public domain dedication).

\section{Grant information}

The author(s) declared that no grants were involved in supporting this work.
1. Chakravarti A, Arora R, Luxemburger C: Fifty years of dengue in India. Trans $R$ Soc Trop Med Hyg. 2012; 106(5): 273-82. PubMed Abstract | Publisher Full Text

2. World Health Organization, Regional Office for South East Asia: Comprehensive guidelines for prevention and control of dengue and dengue hemorrhagic fever: revised and expanded edition. New Delhi, India: World Health Organization; 2011. Reference Source
3. Teixeira MG, Barreto ML: Diagnosis and management of dengue. BMJ. 2009; 339: b4338.

PubMed Abstract | Publisher Full Text

4. Gubler DJ: Epidemic dengue/dengue hemorrhagic fever as a public health, social and economic problem in the 21 st century. Trends Microbiol. 2002; 10(2): 100-3.

PubMed Abstract | Publisher Full Text

5. Garg P, Nagpal J, Khairnar P, et al.: Economic burden of dengue infections in 
India. Trans R Soc Trop Med Hyg. 2008; 102(6): 570-7. PubMed Abstract | Publisher Full Text

6. Sahanaa C, Mishra AK, Bazroy J: Trend of morbidity and mortality of dengue in Tamil Nadu and Puducherry, South India. Int J Community Med Public Health. 2018; 5(1): 322-5.

Publisher Full Text

7. Leo YS, Gan VC, Ng EL, et al:: Utility of warning signs in guiding admission and predicting severe disease in adult dengue. BMC Infect Dis. 2013; 13: 498 PubMed Abstract | Publisher Full Text | Free Full Text

8. World Health Organization and the Special Programme for Research and Training in Tropical Diseases: Dengue Guidelines for diagnosis, treatment, prevention and control: New edition. France; World Health Organization; 2009. PubMed Abstract

9. Premaratna R, Ragupathy A, Miththinda JK, et al:: Timing, predictors, and progress of third space fluid accumulation during preliminary phase fluid resuscitation in adult patients with dengue. Int J Infect Dis. 2013; 17(7): e505-e509. PubMed Abstract | Publisher Full Text

10. Kularatne SA, Weerakoon KG, Munasinghe R, et al.: Trends of fluid requirement in dengue fever and dengue haemorrhagic fever: a single centre experience in Sri Lanka. BMC Res Notes. 2015; 8: 130. PubMed Abstract | Publisher Full Text | Free Full Text

11. Rosenberger KD, Lum L, Alexander N, et al.: Vascular leakage in dengue--clinical spectrum and influence of parenteral fluid therapy. Trop Med Int Health. 2016; 21(3): 445-53.

PubMed Abstract | Publisher Full Text

12. Nasir NH, Mohamad M, Lum LCS, et al.: Effectiveness of a fluid chart in outpatient management of suspected dengue fever: A pilot study. PLOS One.
2017; 12(10): e0183544.

PubMed Abstract | Publisher Full Text | Free Full Text

13. Harris $E$, Pérez $L$, Phares $C R$, et al: Fluid intake and decreased risk for hospitalization for dengue fever, Nicaragua. Emerg Infect Dis. 2003; 9(8): 1003-6. PubMed Abstract | Publisher Full Text | Free Full Text

14. Lopes JA, Jorge S: The RIFLE and AKIN classifications for acute kidney injury: a critical and comprehensive review. Clin Kidney J. 2013; 6(1): 8-14. PubMed Abstract | Publisher Full Text | Free Full Text

15. Viswanathan S: Quantum of fluids in hospitalised patients with dengue and warning signs- a pilot cross-sectional study. Harvard Dataverse, V1. 2019. http://www.doi.org/10.7910/DVN/SKEJAM

16. Nguyen TH, Nguyen TL, Lei HY, et al:: Volume replacement in infants with dengue hemorrhagic fever/dengue shock syndrome. Am J Trop Med Hyg. 2006; 74(4): 684-691.

PubMed Abstract | Publisher Full Text

17. Gopal V, Aruna, Sujatha P: Seroprevalence of Dengue in Antenatal and Paediatric Patients - In a Tertiary Care Hospital, Puducherry. Int J Curr Microbiol App Sci. 2018; 7(6): 667-72. Publisher Full Text

18. Dung NM, Day NP, Tam DT, et al:: Fluid replacement in dengue shock syndrome: a randomized, double-blind comparison of four intravenous-fluid regimens. Clin Infect Dis. 1999; 29(4): 787-94. PublMed Abstract | Publisher Full Text

19. Ministry of Health, Sri Lanka- National Guidelines, in collaboration with Ceylon College of Physicians: Guidelines on management of dengue fever and dengue haemorrhagic fever in adults. Colombo, Sri Lanka: 2010

Reference Source 


\section{Open Peer Review}

\section{Current Peer Review Status: $\mathrm{X}$ ?}

\section{Version 1}

Reviewer Report 02 April 2019

https://doi.org/10.5256/f1000research.19911.r45298

(c) 2019 Srikiatkhachorn A. This is an open access peer review report distributed under the terms of the Creative Commons Attribution License, which permits unrestricted use, distribution, and reproduction in any medium, provided the original work is properly cited.

\section{Anon Srikiatkhachorn}

Institute for Immunology and Informatics, Department of Cell and Molecular Biology, University of Rhode Island, Providence, RI, USA

The manuscript by Ramathan and colleague describes the fluid administration in patients hospitalized for dengue with warning signs (DWS) and evaluate the characteristics related to the amount of fluid received. The important aspect of this work in the "real world" setting where close monitoring of all dengue cases by medical staff may be less than optimum and the patients and relatives play important roles in the primary intervention, namely fluid administration.

Although the manuscript addresses an important issue, there are a number of problems in the methodologies and interpretations. The number of cases included in this report is rather small and the enrolment criteria included those that are not true warning signs. These include comorbidity such as pregnancy, alcoholism, and social reasons. It is not clear how many of cases were enrolled based on these criteria. Further, definitions of severe dengue in this manuscript are different to those according to the WHO 2009 classification and have been expanded to include: a $20 \%$ increase in hematocrit reading, at least 3 fold increase in transaminase or CPK levels. The expanded definitions for warning signs and severe dengue in this manuscript may cause confusions to readers and limit the generalizability of the findings in this report.

Although detailed information regarding staffing of the medical wards was provided this does not clearly convey the measurable indicators of work load and man power utilized for patient care. Measurable indicators such as the ratio of cases to medical and nursing staffs should provide a clearer picture.

Tachycardia was used as an indicator of compensated shock. Tachycardia itself can occur from other reasons including fever, anxiety and discomfort that are not related to volume status. It is not clear whether all cases with tachycardia had been tested for postural hypotension before assigning them as severe.

It is notable that the average amounts of fluid received by dengue cases in this study were 
substantially more than previously reported. A manuscript from Kalaratne et $\mathrm{aI}^{1}$ was referenced to indicate fluid requirement comparable to this study. Upon reviewing this reference, the amount of fluid administered in DF and DHF cases was approximately $3000 \mathrm{ml}$ per day, which is significantly less than the amount given in this current work. Although there were no report of respiratory complications such as pulmonary edema in this series, careful administration of fluid must be exercised to avoid fluid overload and respiratory complications. This also calls into question the accuracy of the estimated amount of fluid in this study.

\section{References}

1. Kularatne SA, Weerakoon KG, Munasinghe R, Ralapanawa UK, et al.: Trends of fluid requirement in dengue fever and dengue haemorrhagic fever: a single centre experience in Sri Lanka.BMC Res Notes. 2015; 8: 130 PubMed Abstract | Publisher Full Text

Is the work clearly and accurately presented and does it cite the current literature? Yes

Is the study design appropriate and is the work technically sound?

Partly

Are sufficient details of methods and analysis provided to allow replication by others? Partly

If applicable, is the statistical analysis and its interpretation appropriate? Partly

Are all the source data underlying the results available to ensure full reproducibility? Yes

Are the conclusions drawn adequately supported by the results? Partly

Competing Interests: No competing interests were disclosed.

Reviewer Expertise: Dengue clinical manifestations, disease mechanisms, and clinical management.

I confirm that I have read this submission and believe that I have an appropriate level of expertise to confirm that it is of an acceptable scientific standard, however I have significant reservations, as outlined above.

Author Response 12 Apr 2019

Stalin Viswanathan, Jawaharlal Institute of Postgraduate Medical Education and Research (JIPMER)., Pondicherry, India

Dear Sir,

The following modifications have been made with regard to your comments for perusal. 
1. As you rightly said, this is a small study and it has been mentioned in the title - a pilot study, partly because of the short duration of the epidemic and a single centre study.

2. All patients were enrolled because of the admitting diagnosis - "Dengue with warning signs". It is only after complete statistical analysis, that some of them have been found to have other features of severe dengue such as postural hypotension or an arrhythmia discovered on ECG

3. The enrolment criteria did not include pregnancy, obesity or alcoholism or diabetes as stated. These factors were considered by the admitting emergency department physician. But the diabetic male and pregnant lady in our study had warning signs. The statement in the manuscript as "warning signs" have been suitably modified.

4. The expanded definitions to include sever dengue had been removed and the original retained. Thus the results section and the tables have been corrected accordingly. Thus hepatitis, myositis and hematocrit increase have been deleted.

5. The nurse-patient and doctor-patient ratio have been added in the section accordingly.

6. As per your comments, tachycardia alone without other symptoms have been NOT considered as compensated shock. Thus the tables with compensated shock and hypotensive shock have been changed. All patients (not only with tachycardia) were checked for postural hypotension on both days.

7. Our patients received more fluids than the one cited in the text, partly because some of them had compensated shock or hypotensive shock along with one or more warning signs noted at the time of admission. Thus they received more boluses and instructions to attendants to serve more fluid drinks. Patients with warning signs are often not checked for postural fall in the emergency department, and similarly, the pulse pressure is often not keenly looked at, and narrowing pulse pressure is often missed. Only in one 30-year-old lady with metrorrhagia, dyspnea, and compensated shock was there a suspicion of fluid overload since she received $10200 \mathrm{~mL}$ on Day 1 and hence only $1750 \mathrm{~mL}$ on Day2. But CXR and USG showed no evidence of either effusion or edema and, therefore she received only RBC transfusions for uterine bleeding.

Regards and thank you.

Stalin V

Competing Interests: None

Reviewer Report 25 March 2019

https://doi.org/10.5256/f1000research.19911.r45296

(C) 2019 Kalayanarooj S. This is an open access peer review report distributed under the terms of the Creative Commons Attribution License, which permits unrestricted use, distribution, and reproduction in any medium, provided the original work is properly cited.

Siripen Kalayanarooj 
Queen Sirikit National Institute of Child Health (QSNICH), Bangkok, Thailand

The authors underscored warning signs (WS) but the definition they used was different from the references ${ }^{1}$ that they cited. Because of the different WS definitions, the enrolment criteria and exclusion criteria were not clear, which resulted in a non-homogeneous study population. They include both shock and non-shock patients, i.e. dengue with WS and severe dengue on the time of admission. So, the results might not be relevant.

In addition, in Table 1 the results revealed that on day 1 , the number of cases with compensated shock increased from 4 (day 1 ) to 7 (day 2 ) in the $<5 \mathrm{~L}$ group and reduced from 7 (day 1 ) to 6 (day 2 ) in the $\geq 5 \mathrm{~L}$ group. The number of cases with hypotensive shock reduced from 7 (day 1 ) to 5 (day 2). Were the shock patients on day 1 and 2 the same patients or different ones?

The same results on day 2: the number of cases with compensated shock increased from 3 (day 1) to 6 (day 2) and number of cases with hypotensive shock increased from 1 to 2 and in the $<5 \mathrm{~L}$ group. In the $\geq 5 \mathrm{~L}$ group, compensated shock reduced from 8 (day 1 ) to 7 (day 2) and from 6 (day 1 ) to 3 (day 2) for hypotensive shock. Were the shock patients on day 1 and 2 the same patients or different ones?

For the above results, even in the $\geq 5 \mathrm{~L}$ group, the amount of fluid didn't seem very effective in maintaining hemodynamic stability. Still, there were more than $50 \%$ of cases with shock, or that developed shock (if not the same patients).

Please clarify number of study cases, 41 (in the abstract) or 43 (in the results and Table 1). Because of different definitions, the number of patients with severe dengue increased, but by looking at the mean platelet count, mean HCT and mean AST/ALT, it seems that the majority of enrolled case were not severe. But in the more severe cases, this fluid amount could not prevent shock in at least 14 cases (33\%) in this study.

The following definitions in this study were different from standard:

Warning signs (WS) that were not in the references: poor oral intake, dizziness, hypotension or shock, tachycardia during the afebrile phase, severe thrombocytopenia, associated diabetes, pregnancy or alcoholism, referral for admission from a peripheral health center and obese patients.

Hypotension was considered when systolic blood pressure was $<100 \mathrm{mmHg}$.

Tachycardia defined compensated shock.

Hypotensive shock = pulse pressure $20 \mathrm{mmHg}$ or systolic BP $<90 \mathrm{mmHg}$ or feeble pulse or the patients was restless.

Organ impairment was defined as:

Transaminase elevation $>3$ times.

CK elevation $>3$ times.

Thrombocytopenia = platelet count, 150,000/cumm.

\section{References}

1. Dengue: Guidelines for Diagnosis, Treatment, Prevention and Control: New Edition. 2009.

PubMed Abstract

Is the work clearly and accurately presented and does it cite the current literature? 
Partly

Is the study design appropriate and is the work technically sound?

No

Are sufficient details of methods and analysis provided to allow replication by others? No

If applicable, is the statistical analysis and its interpretation appropriate?

No

Are all the source data underlying the results available to ensure full reproducibility? No

Are the conclusions drawn adequately supported by the results?

No

Competing Interests: No competing interests were disclosed.

Reviewer Expertise: dengue

I confirm that I have read this submission and believe that I have an appropriate level of expertise to state that I do not consider it to be of an acceptable scientific standard, for reasons outlined above.

\section{Author Response 12 Apr 2019}

Stalin Viswanathan, Jawaharlal Institute of Postgraduate Medical Education and Research (JIPMER)., Pondicherry, India

Dear Sir,

The following modifications have been made by us with regard to your comments for your perusal.

1. The abstract has been corrected to include 43 patients as in the rest of the manuscript.

2. The definitions of warning signs have been included as in WHO 2009 guidelines. The other criteria such as pregnancy and diabetes which are only indicators for admissions and categorizing under Group B have been mentioned separately. nevertheless, both the male diabetic and pregnant lady had warning signs at admission.

3. Tachycardia as a single criterion for compensated shock has been removed.

4. Hypotension has been rewritten as $\mathrm{BP}<90 \mathrm{mmHg}$ and the results have been modified accordingly.

5. Organ impairment with regard to hepatitis has been removed. Since myositis is only part of the expanded dengue syndromes without a clear cut-off level, $3 x$ levels were originally considered significant. But they have been excluded now. Only transaminases and CK levels are mentioned. 
6. Thrombocytopenia has been removed and only severe thrombocytopenia $<50,000$ has been retained.

7. As you have rightly remarked, the majority was not severe. Even the severe cases, were because of having a pulse pressure $<20 \mathrm{mmHg}$, postural drop in BP or hypotension. Patients were taken into the study when the admitting diagnosis was warning signs - they could have had postural hypotension, or they may have worsened further when the study began at $08 \mathrm{AM}$. We did not foresee this, and the true picture of having some severe dengue among the cohort was available only after the statistical analysis.

8. In view of alteration in definitions, the numbers of hypotensive and compensated shock have changed. These have been incorporated, along with the clarification in the results regarding new patients who developed compensated or hypotensive shock. $A$ new row (new compensated shock on Day 2 ) has been added in the table.

Regards,

Stalin V

Competing Interests: None

The benefits of publishing with F1000Research:

- Your article is published within days, with no editorial bias

- You can publish traditional articles, null/negative results, case reports, data notes and more

- The peer review process is transparent and collaborative

- Your article is indexed in PubMed after passing peer review

- Dedicated customer support at every stage

For pre-submission enquiries, contact research@f1000.com 